\title{
Transatlantica
}

Revue d'études américaines. American Studies Journal

\section{Folklore et écriture vernaculaire dans The Autobiography of Miss Jane Pittman}

Au-delà de la couleur locale

\section{Laurence Cossu-Beaumont}

\section{OpenEdition}

Journals

Édition électronique

URL : http://journals.openedition.org/transatlantica/1072

DOI : 10.4000/transatlantica. 1072

ISSN : 1765-2766

Éditeur

AFEA

Référence électronique

Laurence Cossu-Beaumont, «Folklore et écriture vernaculaire dans The Autobiography of Miss Jane

Pittman », Transatlantica [En ligne], 1 | 2006, mis en ligne le 02 mai 2006, consulté le 29 avril 2021.

URL : http://journals.openedition.org/transatlantica/1072 ; DOI : https://doi.org/10.4000/

transatlantica. 1072

Ce document a été généré automatiquement le 29 avril 2021.

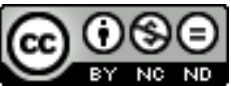

Transatlantica - Revue d'études américaines est mis à disposition selon les termes de la licence Creative Commons Attribution - Pas d'Utilisation Commerciale - Pas de Modification 4.0 International. 


\section{Folklore et écriture vernaculaire dans The Autobiography of Miss Jane Pittman}

Au-delà de la couleur locale

Laurence Cossu-Beaumont

And I wanted to hear that Louisiana dialect-that combination of English, Creole, Cajun, Black. For me there's no more beautiful sound anywhere

[...]. I wanted to read about the true relationship between whites and blacks-about the people

that I had known. (Gaines 1978, 28)

1 C'est ainsi qu'Ernest J. Gaines décrit son projet dans « Miss Jane and I ». Il y évoque le projet d'écrire le "dialecte » de la Louisiane, " anglais, créole, cajun et noir », et le projet d'écrire une communauté, elle aussi métissée. Si The Autobiography of Miss Jane Pittman se lit comme l'autobiographie d'un peuple (« a folk autobiography », Lowe 1995, 94), il convient donc d'envisager avant tout le «son », l'oralité, la musicalité partagée de ce peuple, et la manière dont ce choix d'écriture emmène le lecteur au cœur d'une communauté complexe.

2 Nous aborderons certains aspects du folklore noir qui touchent à l'oralité. Gaines s'inscrit résolument dans cette tradition du conteur, ou encore griot, et rappelle fréquemment que sa source d'inspiration se trouve dans son expérience enfantine des conversations à bâtons rompus sur les marches d'un perron sous les grands arbres de la plantation où il a grandi. A la nostalgie de ces «porch talks » (Lowe 1995, 58) s'ajoute aussi un projet plus ambitieux de témoignage historique et culturel, à l'aune de cette autre référence rappelée par Gaines : « When I wrote Miss Jane Pittman, my Bible was Lay My Burden Down, a collection of short WPA interviews with ex-slaves recorded in the 30's » (Lowe 1995, 94). Gaines reproduit le genre en plaçant le récit sous le signe d'une interview d'une femme qui a vu un siècle passer, enregistrée par un professeur d'histoire ; le magnétophone de l'éditeur (discret après l'introduction) se substitue à la 
plume de l'écrivain (supposément absent de « l'autobiographie ») pour laisser place à la seule voix de Miss Jane (vi-vii). Or nous suggérons que cette voix, son rythme, son humour, sa créativité offrent plus qu'une simple forme de couleur locale. Nous nous proposons de considérer, non pas seulement l'empreinte stylistique de l'oralité qui caractérise le récit de Miss Jane mais aussi les enjeux narratifs qu'elle représente pour l'écrivain, qui est bien sûr tout sauf absent. Comment le style oral vernaculaire de Miss Jane offre-t-il à Gaines une fenêtre sur un mode de vie, une page d'histoire, voire un certain militantisme? L'humour n'est-il pas subversif, le signifyin(g) pratiqué par les noirs américains n'est-il pas forme de résistance, ou du moins de survie?

3 Nous commencerons par rappeler la richesse de la langue noire vernaculaire dont Gaines dote son personnage, richesse qui a contribué à la réussite esthétique et au succès populaire du roman. Gaines se montre virtuose dans cet exercice difficile, voire impossible, que constitue l'écriture de l'oralité. Nous aborderons ensuite la subversion inhérente au folklore vernaculaire. La pratique du signifyin(g) comme stratégie d'opposition permet d'envisager le personnage de Miss Jane non comme une simple conteuse mais aussi comme un trickster dont nous définirons les attributs et l'incarnation dans le récit. Enfin, la citation qui ouvre notre réflexion doit nous permettre de ne pas limiter notre discussion à la seule description de la communauté noire de Louisiane, car si Gaines fonde son écriture sur la langue orale, c'est pour mieux signifier qu'elle est partagée par les autres groupes de la communauté propre à la Louisiane; en ce sens, les "autres voix» qui s'expriment en relais, les "autres histoires » qui se donnent à lire (viii) sont aussi celles des blancs et créoles.

4 Ainsi le projet de « folk autobiography » et sa mise en oeuvre par l'écriture de l'oralité doivent bien être envisagés comme autobiographie d'une certain folklore vernaculaire noir mais aussi comme l'autobiographie, si ce n'est d'un peuple, du moins d'une communauté qui possède un folklore commun et une histoire commune.

Une autobiographie folklorique?

5 Si l'on envisage d'abord le projet de « folk autobiography » dans son sens premier, celui d'une autobiographie folklorique, on court peut-être le risque de réduire l'œuvre à une nostalgique évocation du monde caricatural de la plantation sudiste. En effet, outre l'écueil de produire une pastorale naïve, l'auteur court le risque de proposer une caricature qui rappellerait les «minstrel shows » où l'on se moquait grossièrement de l'anglais forcément inférieur des noirs pour l'agrément du public blanc. Néanmoins la mise en œuvre par Gaines ne laisse pas de doutes quant à la maitrise de l'écrivain de l'art difficile, voire du projet illusoire, de vouloir mettre par écrit ce qui est censé être irréductible à la page figée : le rythme et la musique d'une langue orale qui s'invente sans cesse. Ainsi la simple analyse lexicale et grammaticale que nous réalisons cidessous, qui ne se veut initialement que point de départ méthodologique, révèle déjà la singularité d'un projet esthétique ambitieux, risqué et convaincant, sa réussite exceptionnelle, et ses enjeux politiques.

6 Dans un premier temps, le lecteur ne peut qu'être frappé par une retranscription attendue de certains aspects du dialecte du Bayou qui sont familiers à la langue vernaculaire noire américaine. On note au cours des évocations de Miss Jane l'usage systématique d'irrégularités grammaticales :

- double négation ("You don’t have to say nothing ", vi)

- omission de tout ou partie du verbe (« Oh, Lordy, we free », 11; « Anybody down there with her? », 194) 
- conjugaisons erronées (on notera plus particulièrement la fréquence de « gived » et

«knowed»)

- accords erronés (« We told him some of us was going, some of us was staying »,15) (Je souligne)

- pronoms en place et lieu de déterminants (« Why don’t you take them children back to Kansas ", 111) (Je souligne)

- redondances («The driver he never got in the celebration him »,11) (Je souligne)

Ces marques grammaticales et syntaxiques sont relayées par des effets de prononciations typiques, sous forme de contractions notamment ( I ain't lying, Master ", 7; "He shouldn't'a killed my boy », 130; "What y'all talk about, Mary Agnes? », 178, je souligne). Or c'est dans l'abondance de telles prononciations éloignées de l'anglais standard que se donne à voir (ou plutôt à entendre) la grande créativité de la langue orale. Car, bien plus frappante et innovante que les dites "fautes » susmentionnées et attendues, est la nouveauté d'une musicalité inouïe de la voix de Miss Jane. «I have tried my best to retain Miss Jane's language. Her selection of words ; the rhythm of her speech » (vii). En effet, le prologue place le récit sous le signe de la singularité du discours oral de Miss Jane, qui prime par rapport aux conventions habituelles du dialecte. Gaines souscrit certes à ces conventions, par souci de réalisme, mais il offre bien plus, et là réside son art.

Il existe bien des moments dans le récit où l'oralité joue un rôle capital : le lecteur est littéralement tenu en haleine par certains morceaux de bravoure de la culture folklorique de la «Luzana ». Ainsi l'enchaînement des nouveaux noms que se donnent les compagnons fraîchement émancipés de Jane en route pour le Nord prend la forme d'une cérémonie où sont scandées les nouvelles sonorités de la liberté:

We must have been two dozens of us there, and now everybody started changing names like you change hats [...] This one would say, "My new name Cam Lincoln." That one would say, "My new name Ace Freeman.".Another one would say, "My new name Sherman S. Sherman." (18)

Déjà l'enjeu historique est sous-jacent, sous couvert de virtuosité stylistique, puisque cette scène évoque le moment clé où l'ex-esclave assume sa propre identité et liberté. Gaines a indiqué avoir puisé cet épisode éminemment politique chez Booker $\mathrm{T}$. Washington (Lowe 1995, 70), mais on voit bien comment il se le réapproprie avec délice ici. De la même manière, les enchaînements de latitudes et longitudes, puis d'états et de villes, autour de la lecture de la carte et de l'itinéraire pour l'Ohio $(51,53)$ sont des clins d'œil de l'auteur qui semble s'amuser d'une écriture rythmique tout en rebondissements :

"Yet. I was right," he said. "Yep. Ohio-38, 41 lat ; 80, 84 longi. Iowa, let's see-where you at there, Iowa?-here you is little rascal. Trying to hide from me and I ain't done you nothing, huh? Iowa-40, 44 lat ; 90, 96 longi. Yep, that's where Iowa at, all right. While I'm standing here I might's well look up old Illy. Where you at fellow? Uh-huh, just what I expected-36,43 lat ; 88, 92 longi. Yep, yep, that's it, all right." (51)

“All right, we in Arkansas now. Since Arkansas is North of Luzana, you go'n have to buck back East after you leave Arkansas-that's this way-and you end up in Tennessee. That's right there. All right, now you in Tennessee. You go till you hit Nashville-you done passed Memphis way back here near Arkansas : from Nashville you swing back North again and you ought to find you a good road to Louisvillethat's Kentucky. From Louisville you get to Cincinnati-and you in Ohio." (53) 

plus que la progression de Jane et Ned vers l'Ohio ; on ne peut d'ailleurs attribuer ces comptes rendus géographiques et techniques précis à Jane qui, à l'époque, n'a pas dû en comprendre un mot. Même le lecteur peine à suivre et est invité plutôt à se délecter de la musicalité virtuose de monologues rythmés. Le plaisir du verbe propre à tout écrivain et partagé avec son lecteur est ici mis en œuvre par Gaines sur le mode d'une oralité presque chantée.

11 C'est encore la prononciation qui se trouve au cœur de la stratégie narrative lorsque tel phonème déroutant se trouve mis en valeur. Lorsque Jane et Ned rencontrent, au début de leur périple, le " gov'ment invessagator » new-yorkais aux abords du ferry qu'ils ne peuvent emprunter pour traverser la rivière, celui-ci, avenant et plein de compassion, interroge Ned :

"I say, Little Man, what them rocks for ?" he asked.

"Fiyer,"Ned said. (34)

« Fiyer » est moins familier au lecteur qu'un « ain't » et son isolement dans la réponse de Ned, le fait que cette nouvelle marque orale ne soit pas noyée parmi tant d'autres, dans cette réponse qu'on qualifiera de lapidaire, permet au lecteur de mesurer le poids de la souffrance et la force de détermination du petit garçon. Ces deux pierres, auxquelles Ned, menaçant, s'accroche, sont héritées de sa mère, Big Laura, qui les avait utilisées pour allumer le feu; Jane les lui confie solennellement sur les lieux du massacre (24). Elles le rattachent à son passé perdu et garantissent son salut dans de futurs affrontements. Ce «fiyer » confère donc ici bien plus qu'une couleur locale à la prononciation d'un petit garçon noir du Sud; il œuvre comme outil de caractérisation du personnage, avec la même pudeur que celle qui est propre à Jane et Ned. Le " fiyer » de Ned trouve d'ailleurs sa juste actualisation, quelques pages plus loin, lorsqu'il s'en sert pour frapper le jeune Claiborne qui tente de les lui subtiliser (37). Le « fiyer » sert à exprimer tout ce que le silence de Ned porte en lui : le traumatisme de la mort violente de sa mère et le courage de ces deux enfants (Jane n'a pas plus de quatorze ans) qui survivront coûte que coûte.

13 Cette dextérité avec laquelle Gaines se sert du parler vernaculaire noir pour discrètement transmettre des éléments narratifs comme la caractérisation des personnages ou pour allègrement partager le pur plaisir d'une langue créative et musicale a été relevée par le critique Clarence Major, bien connu pour son Juba to Jive (1994), ouvrage de référence sur la langue orale noire :

The result is a controlled, artful story; and, what is equally important, its dramadialogue and narration-are delivered through its syntax rather than through the cheap tricks of written dialect that too often plague our fictionists and poets. (Major 1971, 8)

En plus des effets de sonorités du dialecte, Gaines s'attache en effet à tisser les marques de l'oralité dans l'écheveau même de la narration, par la syntaxe notamment, comme c'est le cas des exemples d'écriture relatifs à la carte ou au "fiyer » que nous venons d'analyser. Pour Major, cet usage même du folklore est la clé de la réussite esthétique, mais aussi politique, du roman :

Gaines' method avoids the obvious pitfall of social propaganda by a relentless use of Black folklore along with and throughout Miss Jane's narrative. In addition, Gaines does not impose on us, or his story, ideological concepts. However, he never permits us to forget the oppressive social and political framework in which Black people, as seen through Miss Jane's eye, move and struggle [...] Whether or not one 
chooses to believe that a novelist should try to solve social problems through fiction, there simply is not any evidence that social propaganda has ever helped anybody toward a larger or deeper vision and sense of life. On the other hand, real art, such as this book represents, does achieve this goal. Real works of art stand up and prove themselves over and over. We may be sure and pleased to remember that an artist such as Ernest J. Gaines is, in the most important -though perhaps not in the most accessiblesense, our true revolutionary. (Major 1971, 8. Je souligne)

L'autobiographie d'un folklore subversif

Revenons sur cette suggestion apparemment audacieuse de Clarence Major : l'œuvre serait "révolutionnaire". Le raccourci étonne mais il permet de revenir sur une dimension fondamentale et cachée du folklore noir américain qui trouve sa juste expression à travers la voix de Miss Jane: son militantisme et sa subversion, en particulier à travers l'art du signifyin(g) ; traduit mot à mot, c'est l'art de « faire le sens » (ou plutôt de le cacher). Henry Louis Gates Jr. rappelle dans The Signifyin(g) Monkey (1988) les multiples définitions de cette pratique orale noire américaine : on retiendra qu'il s'agit d'une rhétorique mettant en œuvre l'humour, la parodie, la moquerie, le sous-entendu, le jeu de mot, le mensonge, dans le but de dénoncer, de duper, de railler un adversaire qui peut être complice ou victime (Gates 1988, 75). Cette tradition trouve son origine dans la légende populaire du singe qui trompe le lion en lui faisant croire que l'éléphant l'a insulté; le lion, qui prend le singe au premier degré, exige des excuses de l'éléphant; ce dernier non seulement les lui refuse puisqu'elles n'ont pas lieu d'être, mais lui inflige une correction pour son impertinence. Le lion est puni de n'avoir pas su saisir le discours figuré, et non littéral, du singe. Gates conclut: "The Monkey's trick depends on the Lion's inability to mediate between these two poles of signification, of meaning " (Gates1995, 55). Ainsi ceux qui ne sont pas initiés aux codes du signifyin(g) sont incapables de dépasser le sens littéral du discours; le sens figuré est reconnaissable pour les seuls initiés. En d'autres termes, il est possible pour les noirs d'utiliser en présence des blancs cet outil qui est rhétorique du détournement, de la duperie virtuose, et d'exprimer des idées contradictoires sans se mettre en danger. L'humour devient source de pouvoir pour les noirs dans un contexte socio-politique où ils n'en ont aucun - si ce n'est celui du verbe: ils peuvent donner l'apparence de l'obéissance, de la bêtise, de l'amusement innocent tout étant en train d'exprimer une plainte ou une protestation.

16 Le passage où Jimmy offre à la plantation une représentation d'un " minstrel show » en est un exemple éloquent (227). Ce genre de spectacle où les acteurs noirs s'enduisaient le visage de cirage noir pour grossir encore le trait de leur différence et s'autocaricaturaient au long de répliques et sketches écrits par des blancs, pour le plaisir d'un public blanc avide de rire du ridicule des noirs, est repris par Jimmy devant un auditoire noir. Il s'agit donc déjà d'une réappropriation par des noirs d'un élément d'oppression blanc. En effet, Jimmy y imite, non pas la façon dont parlent les noirs, mais la façon dont les blancs caricaturent les noirs. Lors de ce spectacle, on rit donc des blancs et non des noirs, tout en donnant l'impression, de l'extérieur, de se soumettre parfaitement innocemment à un genre de distraction blanc. On rit donc de ce qui fait souffrir, exercice récurrent dans les conversations noires. Joe Brown et ses collègues, qui font face à la mort chaque jour en domptant, pour des blancs bien à l'abri, des chevaux qui font la fortune de leurs maîtres, n'ont d'autre stratégie que le rire pour échapper à la peur qui les tenaille et au pressentiment funeste de Jane : «While he was back there Joe told him what I had said about the horse, and everybody burst out 
laughing [...] Joe laughed so much he cried» (94). Le rire à la place des larmes: l'humour et la dérision sont la seule stratégie de survie.

On se protège avec l'humour, mais on peut aussi combattre. «When she spoke she used as few words as possible. Yet, there were times when she would repeat a word or phrase over and over when she thought it might add humour or drama to the situation » (vii). Souvent, cet humour calculé de Jane est habilement dirigé contre quelqu'un. De fait, l'aïeule riche de l'expérience d'une vie semble avoir acquis une certaine propension à l'ironie dévastatrice. Lorsqu'elle évoque les discours belliqueux et moralistes des Sudistes va-t-en-guerre contre les abolitionnistes mécréants, elle ajoute sous forme d'une parenthèse :

"Don't put my food up," they said, "Don't put it up and don't give it away. I'm go'n kill me up some Yankees and I'm coming right back home. Who they think they is trying to destruck us way of living? We the nobles, not them. God put us here to live the way we want to live, that's in the Bible" (I have asked people to find that in the Bible for me, but no one's found it yet). (4)

Le rappel ironique de la confiance aveugle des Sudistes, à travers un effet d'exagération manifeste ("Don't put my food up »), sonne comme une cruelle moquerie à l'égard d'une population dont la défaite fut d'autant plus cuisante qu'elle était, pour eux, inattendue. La fragilité des justifications « spirituelles » n'est pas formulée directement mais à l'occasion d'un aparté supposément objectif. C'est au second degré que Miss Jane fustige les soldats de la guerre.

Mais cette maîtrise du folklore noir et de ses formes d'expression n'est pas l'apanage de la vieille femme expérimentée. Tout au long du récit et de sa vie, la frêle Miss Jane, avec sa vivacité et son fort caractère, use et abuse de la pratique du signifyin(g) contre les Samson qu'elle rencontre sur son parcours. Dès son jeune âge, elle affronte avec audace des adversaires plus forts qu'elle. A l'intérieur de la communauté noire, où ces rites sont connus de tous, la jeune Ticey défie le contremaître noir qui la rappelle à l'ordre le jour de l'émancipation :

The driver said: “Shut up. You ain't nothing but trouble. I ain't had nothing but trouble out you since you come in that field."

"If I ain't nothing but trouble, you ain't nothing but Nothing," I said.

And the next thing I knowed, my mouth was numb and I was laying down there on the ground. (11)

Le contremaitre incarne une figure à mi-chemin entre les blancs qui lui ont transmis leur autorité, autorité dont il abuse en reproduisant le discours qu'un maître blanc pourrait tenir à Jane dans ce contexte, et les noirs dont il connaît et partage la tradition et les pratiques orales. Ainsi, il se sait battu par la réplique brillante de Jane. Dans une joute verbale du signifyin(g), la victoire est remportée par celui qui réduit son adversaire au silence. Or le contremaître est incapable de renchérir: quelle insulte pourrait surpasser le fait de n'être "rien » ? Il doit avoir recours au stratagème de ceux qui sont forts physiquement mais faibles verbalement : il frappe Jane et l'envoie au sol. Mais ce geste apparemment triomphal est en fait un aveu de son impuissance à contrôler l'esprit de Jane, à réduire son indépendance farouche, et un aveu de sa défaite publique (devant le maître et les autres ex-esclaves). A ce point du roman et de l'Histoire, la mésaventure du colosse face à la petite Jane est emblématique de la perte totale de son statut privilégié et de son autorité incontestée qui accompagneront irrémédiablement la fin de l'esclavage. 
21 Au cours du récit, on mesure à quel point Miss Jane, en tant que conteuse, est aussi maître du sens de son histoire, et pourquoi pas maître de l'Histoire. Dans la tradition vernaculaire noire, le personnage du singe est non seulement capable de manipuler adroitement le lion par des insinuations adroitement choisies, mais aussi de "créer " l'événement puisque, sans son intervention, il n'y aurait pas de bagarre entre le lion et l'éléphant. A cet égard, Miss Jane est aussi «trickster», soit cette figure de l'illusionniste du verbe capable de " changer » la réalité des choses. A la fin du récit, en particulier, Miss Jane assume le rôle de guide qui emmène la communauté vers son destin de rébellion politique. Mais ce n'est pas seulement son geste (se mettre en marche vers Bayonne où Jimmy doit être soutenu dans son combat) qui permet l'initiation et la poursuite de la lutte. Son récit du départ pour Bayonne au petit matin, pourtant simple compte rendu d'un déplacement à travers la plantation encore endormie, offre un jeu d'échos entre les verbes «to walk/march" et "to stand». Quand on sait que le signifyin(g) joue sur le sens littéral d'un mot et son sens figuré, on ne peut qu'admirer le double sens de chacun de ces verbes anodins. Miss Jane ne fait rien de plus que traverser la plantation à pied (to walk), mais ce geste même est militant (to march). Les autres habitants ne font que se tenir à ses côtés (« But they was standing there, and that's what mattered »,257), mais cette simple présence est comme un poing levé (ne dit-on pas " to stand up for one's rights»). Ce jeu lexical propre à la tradition du signifyin(g) orchestré par Gaines ne manque pas de rappeler la dédicace sur laquelle s'ouvre son roman :

To the memory of

My beloved aunt, Miss Augusteen Jefferson,

who did not walk a day in her life

but who taught me the importance of standing

Et lorsque tout semble fini, lorsque l'heure de gloire apparaît vaine avec l'annonce de la mort de Jimmy, c'est encore en jouant avec les mots, en jouant sur les mots que Miss Jane encourage les siens à ne pas renoncer.

"Jimmy is dead," Robert said [...]

"Just a little piece of him is dead," I said. "The rest of him is waiting for us in

Bayonne." (259)

C'est en ce sens que Gaines tisse le militantisme discret de son personnage, en hommage aux modestes artisans de la lutte des droits civiques qui surent croire en l'improbable, dans la maille même du récit de sa narratrice et porte-voix.

Folklore, histoire et héritage d'une communauté métissée

On a longuement parlé de cette stratégie noire: lutter, voire créer et changer les choses, par le verbe. Or Gaines indique bien dans son projet son souci de faire honneur aux sonorités de toute une communauté, riche de différents groupes: noirs, blancs, créoles. A cet égard, on ne peut s'arrêter à des considérations sur le folklore noir dans The Autobiography of Miss Jane Pittman, car ce folklore est partagé par toutes les composantes de la bien singulière société de «Luzana ».

Les blancs qui entourent Miss Jane ne parlent pas non plus l'anglais standard. Dès les premières pages, sa première maîtresse, avec qui elle est loin d'être complice, s'exprime dans une tonalité proche de celle de Miss Jane: "What you standing there for?» (5). Bien des années après, le propriétaire de la plantation où Miss Jane finit sa vie, Robert Samson, et son épouse Miss Amma Dean ont les mêmes accents. Lorsqu'ils tentent de convaincre Miss Jane de ne pas quitter la grande maison, ils parlent avec elle de «lectwicity", participant à la créativité presque musicale des noirs du sud. Les 
répliques de Robert Samson portent les mêmes marques syntaxiques et phonétiques que nous avons signalées chez les personnages noirs : "I said nothing "bout no pen" (199). Tous les personnages blancs rencontrés par Miss Jane dans son périple du début, puis durant sa longue vie, s'expriment sur un mode similaire, qu'ils soient de milieux modestes ou aisés. Mais, plus intéressant, ils partagent aussi la culture folklorique des noirs ; du moins ont-ils quelques points communs dans leur manière de réagir avec un humour grinçant à certaines situations de crise. Ainsi, lorsqu'elle finit par affirmer son refus de rester plus longtemps vivre chez les Samson et s'interroge sur le choix de la maison qui sera désormais la sienne sur la plantation, Miss Jane demande :

"Mr. Robert, is it all right with you if I moved in that house with Mary ?"

"You asking me ?" he said. "I didn't know I still running Samson. I thought you was.

I thought it was up to you to tell me when you wanted to move and where. And it

was my duty to go there and clean up the place for you." (213-214)

La diatribe de Robert Samson continue sur une dizaine de lignes supplémentaires. Il fait preuve ici d'une ironie qui lui permet à la fois de remettre Miss Jane à sa juste place, même si l'entreprise est vaine tant l'aïeule est connue pour son caractère irascible, mais aussi d'exprimer sa contrariété face à ce départ définitif du foyer des Samson. Autorité et pudeur sont chez lui aussi au cœur de stratégies verbales jouant sur le double sens de ses affirmations. Ce ton ironique est tout à fait évocateur de la réponse de Miss Jane au professeur d'histoire qui vient l'interviewer. Il n'acceptera aucun refus de sa part, lui signifie-t-il avec respect : "Then I reckon I better say something (v). Faire mine de se soumettre à l'autorité, tout en réaffirmant son propre pouvoir, pour céder sans en avoir l'air : une même réponse et un même ton pour Robert Samson et Miss Jane.

Noirs et blancs, maîtres et (ex-)esclaves partagent donc un folklore en partie commun. Celui-ci est le ferment d'une histoire commune, qui, puisqu'ils partagent certains modes d'expression, peut aussi s'écrire en commun. Un des moments forts du roman réside dans la mort tragique de Tee Bob qui illustre le gouffre qui sépare les différentes composantes de la société de Louisiane. Il est impossible à un jeune blanc de la bonne société d'envisager d'épouser une jeune femme d'origine créole comme Mary Agnes. Il peut avoir une aventure - c'est même dans l'ordre des choses, comme son propre père a eu un enfant métisse (Timmy) - mais non l'aimer, et surtout pas l'avouer en public. Son ami Jimmy Caya se fait le relais des tabous absolus transmis de générations en générations :

"Robert," he told him. "Don't you listen in class? Ain't you heard him » (I forget that teacher's name, but I think he said Gamby) "over and over and over? You think it's changed since then? She's the same woman, Robert. She know her duty, and all she expect from you is ride the horse down there. But that's far as she expect you to go. The rest is her duty, Robert. She knows that. He" (I'm almost sure he called that teacher Gamby) "told you it was like that then, and it's the same way now." (182)

Mary Agnes est elle-même issue de la bonne société créole métissée, fruit d'alliances secrètes et tues : sa grand-mère fut la compagne d'un homme blanc, M. LeFabre, qui lui légua sa fortune (« even slaves », 166). La complexité de son identité pousse Mary Agnes vers un destin inhabituel; elle retourne à ses origines en décidant de se consacrer à l'éducation d'enfants noirs ("And for the rest of her life, Mary Agnes was trying to make up for this : for what her own people had done her own people», 166). Sa famille et sa communauté rejettent ce choix contraire aux conventions : "When Mary Agnes came here to teach school, the people at Creole Place told her never come back home 
again » (170). Les préjugés et tabous raciaux sont donc reproduits à plusieurs niveaux entre les différentes castes sociales et raciales de Louisiane, qui pourtant vivent ensemble et souffrent ensemble (le père de Mary Agnes, après les menaces, finit par la supplier à genoux de rentrer chez eux, 170). Le drame qui se noue alors, avec le suicide tragique de Tee Bob, qui meurt du poids des traditions ancestrales et interdictions raciales (il se tue avec le coupe papier de son grand-père, dans le bureau, sous le regard des portraits de ses aïeuls, 195-6), est le drame collectif d'une communauté à la fois une et déchirée ("We all killed him », 201). Mais, à cette occasion, la communauté réagit avec moins d'hypocrisie et de haine que de coutume, du moins dans l'intimité, sous l'impulsion de Jules Raynard. Le personnage est, dans cette scène, comme le double de Miss Jane puisqu'ils ne se quittent pas et commentent ensemble les raisons du drame. Robert Samson est mis face à ses contradictions ; il renonce à se venger de Mary Agnes, qui en d'autres temps aurait encouru une mort certaine. Ainsi, c'est un blanc de la bonne société, Jules Raynard, qui apaise les tensions raciales qui auraient nécessairement dû éclater, et c'est avec cet homme blanc que Miss Jane partage momentanément son récit, un récit à deux voix de la dernière conversation de Mary Agnes et Tee Bob avant son geste fatal :

"But ain't this speculatin'?" I said.

"It would be speculatin' if two white people was sitting here talking," Jules Raynard

said, looking round [...]

"But it's us?" I said.

"And that makes it gospel truth," he said. (205)

29 Ainsi, la reconstitution de cette scène-clé pour la transmission du drame, qui est aussi écriture de l'histoire de la communauté, est commune ; elle réconcilie symboliquement les communautés qui vont posséder la même histoire, et non une version chacune aux dépens de l'autre. Récit blanc et récit noir sont portés par la même tradition folklorique de la reconstitution orale des événements marquants de la communauté. A l'inverse, Miss Jane, qui a accueilli et partagé l'initiative de Jules Raynard, refuse le mode d'expression d'un personnage qui est pourtant noir. L'ami que ramène Jimmy à la plantation et qui est son compagnon de lutte ne connaît visiblement pas le ton adéquat pour s'exprimer dans la communauté. Miss Jane le rejette immédiatement sur le critère de son mode d'expression pompeux et de ce fait suspect : " "Well I can do without your retrick here," I said. "If you can say nothing sensible, don't say nothing" " (243). La langue parlée peut donc rapprocher blancs et noirs, lorsqu'ils en partagent les rites, et peut se poser en barrière entre les noirs si elle ne leur est plus commune. Grâce à ces moments inattendus d'harmonie ou de divorce entre des personnages que tout est censé séparer ou qui se trouvent au contraire étonnamment déconnectés, Gaines transmet toute la complexité d'une société métissée aux rapports ambigus et complexes. Il a chaque fois recours à un discours partagé ou un discours divergent : l'usage subtil et à de multiples niveaux de la tradition orale dans l'écriture de son roman lui permet de représenter les «vraies relations » entre noirs et blancs qu'il évoque dans notre citation initiale.

30 A la fin du roman, ce sont encore Jane et Robert qui sont au cœur de cette communauté multiple à travers leur relation complexe et paradoxale. Le maître qu'il reste s'efface parfois devant la compréhension silencieuse qu'il partage avec Jane. A travers leurs caractères similaires, leur complicité verbale, les deux personnages servent d'emblème à la complexité de la communauté métissée de Louisiane. La dernière phrase du roman en est la juste expression - expression silencieuse : «Me and Robert looked at each 
other there a long time, then I went by him » (259). Rien n'est prononcé, tout est dit, dans ce dernier regard défiant; le destin de la communauté noire est scellé sous les yeux impuissants mais lucides des blancs. La fin du roman n'est que le début d'une nouvelle ère.

\section{BIBLIOGRAPHIE}

BOTKIN, B.A., (ed), Lay My Burden Down. A Folk History of Slavery. Athens : University of Georgia Press, 1989.

GAINES, Ernest J., The Autobiography of Miss Jane Pittman. New York : Bantam Books, [1971] 1972. « Miss Jane and I », Callaloo, N³, Ernest J. Gaines : A Special Issue, May, 1978, 23-28.

GATES, Henry Louis Jr., The Signifyin(g) Monkey, A Theory of African American Literary Criticism. New York \& Oxford : Oxford University Press, 1988.

LOWE, John, (ed), Conversations with Ernest J. Gaines. Jackson : University Press of Mississippi, 1995. MAJOR, Clarence, Essence, September 1971.

MAJOR, Clarence, From Juba to Jive : A Dictionary of African American Slang. New York : Penguin, 1994.

\section{RÉSUMÉS}

On commencera par relever la richesse vernaculaire de la voix de Miss Jane et la densité folklorique de l'écriture de Gaines qui décrit ainsi son projet: "a folk autobiography ». Une autobiographie «folklorique » ? Certes, le rythme, la grammaire de l'anglais oral noir américain jalonnent ce récit qui se place lui-même dans son prologue sous le signe stylistique des conversations informelles sur les marches d'un perron de Louisiane, ces «porch talk» dont Gaines répète s'être inspiré.

Cependant, l'analyse confinerait à la couleur locale si la "folk autobiography » n'était pas lue comme autobiographie fictive d'un peuple et d'une stratégie de survie : Miss Jane incarne aussi la dimension fonctionnelle du parler vernaculaire dont la théorie de $\operatorname{signifyin}(g)$ a montré les enjeux vitaux. La jeune Jane, tout juste libérée de son nom d'esclave, possède déjà les " armes » verbales pour attaquer une société vacillante. Elle parvient à "éliminer" un adversaire des plus symboliques, le contremaître noir, en le réduisant à néant dans une réplique emblématique du pouvoir du signifyin(g) («You ain’t nothing but Nothing» p. 11). La veille femme, sage mais non résignée, devient trickster à la fin du récit. Dans une évocation performative (on notera le jeu des « to stand ", « to march » p. 257), elle fait plus que « raconter » son histoire et parvient à « créer » l'Histoire en guidant et modifiant le destin de toute une communauté.

On suggérera encore que cette "folk autobiography", autobiographie d'un peuple ou d'une culture, ne se laisse pas réduire à la seule communauté noire. Le récit est ancré dans la culture de la Louisiane, une culture complexe où maîtres blancs et esclaves puis métayers noirs traversent 
des tragédies communes, et écrivent ensemble leur Histoire commune dans une langue vernaculaire qu'ils partagent aussi, ainsi que l'illustre le drame de Tee Bob et Mary Agnes, et surtout le récit à deux voix qu'en font Jane et Jules Raynard (« speculatin » p. 205).

\section{INDEX}

Keywords : folklore, rhetoric, autobiography, Louisiana, community, orality, history, voice, Gaines Ernest J., politics, interraciality, aesthetics, humour, narration, signifying, trickster, cultural mixing, dialect, irony, literalness, vernacular

Mots-clés : rhétorique, autobiographie, Louisiane, communauté, oralité, histoire, voix, politique, interracialité, esthétique, dialecte, ironie, littéralité, métissage, vernaculaire

\section{AUTEUR}

\section{LAURENCE COSSU-BEAUMONT}

Laurence Cossu-Beaumont est Maître de Conférences à l'Université de Picardie. 